so dangerous to make positive statements as to the efficacy of a particular medicinal agent.

The pages of this Journal have repeatedly referred to the uses of bromide of potassium in epilepsy. Statements in its favour have been contradicted by writers holding different views. The abstract question as to the value of bromide of potassium in epilepsy was put, as if the disease were a simple perversion of a normal function or a single well definable lesion, which could be neutralised by a given antidote. We have scarcely arrived at a sufficiently precise knowledge of the disease as yet, to reduce the question of treatment to so simple a denomination. We do not know nearly as much of epilepsy as we do of pneumonia, as to the local changes occurring in the body; but, although our knowledge is almost limited to the external phenomena, we are sufficiently advanced in our nosology, to affirm that the circumstances influencing and giving rise to the disease are as complicated as in any disease in the most complete nosological list. It would accordingly be scarcely fair, either to the disease or to the remedy to be tested, to take at haphazard a given number of cases characterised by the epileptic paroxysm, and to determine by the result whether or not the remedy deserved our confidence.

My objections to such a procedure would become still greater, if I were told that the cases were all of considerable standing, and justified the assumption that secondary cerebral lesions had already resulted.

We know of bromide of potassium, that it may be taken in large doses with impunity, and that it passes off by the kidneys; but we know nothing definite of its action on the nervous system. It behoves us, therefore, to be extremely careful not to reject an agent on insufficient grounds; but it is not so much my object to advocate the use of this agent, as to suggest that it does not follow that it is useless because in an asylum containing many confirmed epileptics it fails to produce the desired effects.

I am a firm believer in the general use of drugs in the treatment of disease; but I am habitually sceptical as to the effect produced by any given remedy in an individual instance, knowing the limitation of our powers of observation and analysis. I am not likely, therefore, to over-estimate any medicine; but, unless I have altogether wasted my time and opportunities, I have satisfied myself that there are many cases of epilepsy in which medicines not only benefit but cure the patient; and I believe, also, that in some-alas! but too few-it was something more than a mere coincidence that bromide of potassium arrested the disease. I am, however, equally certain that there are a large number of cases in which it is useless; and that epilepsy, like all other diseases, must not be treated as an unity, but regarded in all its aspects, and treated according to the various phenomena under which it may present itself. Bromide of potassium may be useful in one class of cases, but not in others ; just as nitric or nitrohydrochloric acid benefits a certain class, and is useless or injurious in others. The iodides, again, are undoubtedly beneficial in some forms of the disease. Chalybeates, again, are required by some, and aggravate the malady in others.

It is not my wish, nor do I know that I could, with satisfaction to myself or the readers of the JouRNAL, classify the different forms, with the precision that I believe to be necessary; but of this I feel assured, that if we are to arrive at any definite and scientific result with regard to remedies in this particular disease, we must not lump all our cases together, and treat them all with the same remedy, especially with one the physiological action of which is as yet so little determined as that of bromide of potassium.

\section{ON THE NATURE AND TREATMENT OF FEVER.}

By A. B. Steele, EsQ., Liverpool.

[Read at the Liverpool Medical Institution, February 9th, 1865.]

I HAVE long been impressed with the conviction that the study of fever (especially with reference to its therapeutics) as pursued in the systematic treatises of authors, differs materially from the study of the disease as read in the book of nature at the bedside. I am disposed to attribute the confused, unsatisfactory, and conflicting conclusions to which the written history of fever leads the inquirer, to two principal errors:- - first, that authors have laboured to establish a method of cure; and secondly, that they have based their systems upon a preconceived theory rather than upon clinical observance of the phenomena of the disease. To one or both of these mistakes, may be referred the erroneous but now happily exploded doctrines of Hamilton, Armstrong, Broussais, Clutterbuck, and others; it also explains the ephemeral reputation of those highly vaunted specifics, such as cold effusion, yeast, mineral acids, quinine, phosphorus, antimony, chlorine, mercury, and even alcohol, most, if not all of which, especially the latter, may be useful as remedies under certain circumstances, when given on rational principles, but all of which infallibly become doubtful or dangerous when regarded as specifics. Indeed I hope to be able to show that not only is there no specific for fever, but that, as Dr. Corrigan has observed, in the present state of our knowledge there can be no specific for this disorder.

In Dr. Watson's Lectures, we find the following allusion to the opinion of Pitcairn on this subject. "I do not" (says he) "like fever-curers; you may guide a fever; you cannot cure it. What would you think of a pilot who attempted to quell a storm? Either position is equally absurd. In the storm you steer the ship as well as you can; and in a fever you can only employ patience and judicious measures to meet the difficulties of the case." (Vol. ii, p. 843).

This sagacious and profound remark, in my opinion, comprises the whole principles of fever therapeutics. Although it guards us against useless interference and injudicious meddling with the operations of nature, it by no means sanctions or justifies a mere "do nothing" system, which is sometimes disguised under the specious title of " expectant treatment."

Without dwelling upon the now almost discarded doctrines of physicians of a former generation, I propose to point out what seems to me a want of practical soundness in the teaching of some authors of our own time. Let us appeal to Dr. Murchison, whose treatise on fever is considered one :of the best and most recent text-books, and which undoubtedly contains a fund of valuable information on the subject of which it treats. In criticising the opinions of a physician of high repute and acknowledged ability, I must be allowed to say, that I do not for one moment venture to question the soundness or the success of his practice. It is the doctrines enunciated in his writings, and the inferences he deduces therefrom, to which I take exception, as calculated to confuse and mislead rather than to guide and assist the inexperienced and inquiring practitioner. Dr. Murchison's theory may be summed up as follows. (Pp. 15-16.)

1. The fever-poison enters the blood.

2 . The nervous system (and particularly the sympathetic and vagus) is paralysed.

Here we at once detect what seems to me an important fallacy. In many cases of fever it is not paralysis, but the very reverse; namely, exalted or 
unduly excited action of the nervous system which constitutes the predominant characteristic of the disease, as exemplified by the condition which has been designated "delirium ferox." Dr. Murchison completes his definition by particularising various morbid processes such as increased retrograde metamorphosis of the tissues, non-elimination, and so on, which are doubtless in accordance with sound pathology, but which, nevertheless, are after all too remote and perhaps too hypothetical to lead to a clear and safe basis for treatment, when compared with the conclusions drawn from clinical observation of the phenomena of the disease.

The indications which Dr. Murchison founds upon his theory are: to neutralise or to eliminate the poison, followed by other directions, which $I$ have no difficulty in accepting as in accordance with sound practice, and which, therefore, require no special notice here; but I maintain that to send a student or an inexperienced practitioner to treat a case of typhus fever, with this notion impressed on his mind-namely, that his primary object is to neutralise or to eliminate a poison-is calculated to lead him altogether in a wrong direction. He would naturally first inquire, what is the nature of the poison, what its antidote, or by what special emunctory it is to be eliminated?

Like all doctrinaires, our author has his answer ready : "Typhus poison" (Murchison On Fever, p. 116), he tells us, "is some unknown compound of ammonia" (ibidem), and although he adds that it is perhaps premature or rash to hazard a conjecture as to its exact nature (p. 114); yet he founds upon this theory the use of mineral acids as neutralisers of ammonia in the blood (vide p. 265). A prominent direction for treatment, given by the author I am quoting, is, that "elimination is to be encouraged by maintaining the action of the kidneys, the bowels, and the skin." (P. 267.)

Now, I am at once prepared to admit that, under the skilful direction of the author himself, these indications may be, and no doubt are, so judiciously carried out, as in no way to interfere with the favourable progress of his patients; but to my mind, his theory is opposed to the teaching of clinical experience and to the common sense view of treatment which experience dictates. We have nothing approaching to a well founded record of the cure or arrest of typhus fever by the neutralisation of ammonia, or by the destruction of any other animal poison; and as to its elimination by purging or diaphoresis, this doctrine is opposed to the practice of the most experienced physicians of the present day-not excluding even our author himself-for it is but doing him justice to point out that in his directions for treatment, his practical experience, as I. have already suggested, modifies or corrects the fallacious tendencies of his theories.

Purgatives in typhus, except in mild doses, and most cautiously and sparingly given, have been condemned by Graves, Corrigan, and others ;* and as to diaphoretics, these remedies are as a rule contraindicated in typhus fever, for, as Dr. Corrigan has pointed out, " $a$ crisis by perspiration is of all forms that which is most to be dreaded in maculated fever "I cannot," he adds, "explain this, but of the fact I have no doubt. I have such a dread of crisis by perspiration, that I would much rather see a case of

* Since this paper was written, the following remarks have ap peared from Dr. Peacock of St. Thomas's Hospital. "In all cases of fever, I am especially careful to avoid purgatives in the early stages; and if snme aperient must be given, employ those which are of the mildest character.". "I have frequently seen, both in typhus and typhoid, diarrhos established by the injudicious employment of purgatives, which it has been difficult or impossible to arrest." (Lancet, Feb. 11th, 1865, p. 146.) maculated fever considerably prolonged, than that the risk of crisis by perspiration should be incurred." Dr. Tweedie thus records his opinions on this point : "Insufficient grounds have been adduced for the employment of diaphoretics, which appear to have been suggested by observing the occasional termination of acute diseases by sweating. No one, however, I believe, ever witnessed a single case in which there was the smallest grounds for believing that the fever-poison was destroyed by such means." (Lec tures on Fevers, p. 209.)

Dr. Murchison himself says: "After an extensive use of these remedies (diaphoretics), I cannot say that I have obtained very satisfactory results from them. Even when diaphoresis is produced, there is no necessary improvement in the general symptoms, while profuse sweating is often followed by increased prostration." With reference to elimination by the kidneys, a due consideration of the condition of the organs of the body, which we shall more particularly notice hereafter, renders it extremely probable that, in many cases, remedies which directly stimulate the kidneys would tend to increase congestion of those organs, rather than to excite them to increased action.

The fallacy of the eliminating mode of treatment is rendered still more apparent by reference to another disease, which has many points of analogy with typhus; I mean small-pox, which, like typhus, is the result of an animal poison introduced into the blood, has a specific eruption, and runs a definite course. No physician of the present day would, I presume, attempt to treat small-pox on the principle of elimination; the same may be said of measles, scarlatina, and other analogous febrile diseases. The conclusions to which the prolonged and extensive experience of Dr. Tweedie on this point has led him, is thus expressed in his own words : "It is apparent that fever when once developed can rarely be arrested; or, in other words, that the means of expelling the poison, or of depriving it of its noxious effects, have yet to be discovered. The duty of the practitioner, therefore, is to endeavour to guide the disease, and to prevent as much as possible injury to organs essential to life." (Op. cit., pp. 211-12.)

Let us turn for a moment to another modern author of high repute, namely, Dr. Watson. His Lectures on the Practice of Physic, excellent as they are in all other respects, have nevertheless this objection, that fever is divided into weekly periods. This method of treating the subject presents a weak point; namely, that although from the nature of the case it will very often-perhaps most often-coincide with actual observation, yet when it is right, it is only right by accident, and although applicable to a large proportion of cases, may often also lead to a wrong conclusion in practice. The treatment of fever, as we shall presently see, is not to be regulated by the circumstance of its being the first, second, or third week of its duration, but by the state of each particular function day by day. "One case of fever may require as much wine on the second day of its attack, as another on the twentieth; and the bleeding or leeching that will not be borne in one case on the third day, will benefit another after a lapse of many days." (Dr. Corrigan On Fever, passim.)

There is another mode by which attempts have been made to lay down the principles of the treatment of fever; namely, by statistics, which appears to me most fallacious and untrustworthy. The ever varying rate of mortality from typhus at different periods of life, and during different epidemics, deprives that mode of calculation of much reliable value in determining the question of treatment, except on a very broad and general interpretation. 
This point is illustrated by the following record of fever statistics from Dr. 'Tweedie's lectures. The death-rate in the London Fever Hospital was 7 per cent. in 1851, 9 per cent. in 1862, and 20 per cent. in 1848. In Aberdeen it was 4 per cent. in one year, 18 per cent. in another year. In the Dublin hospitals for the last forty years, it has never reached 10 per cent. In Cork, the average was about 6 per cent. (Op. cit., p. 200.)

The influence of age is shown by Dr. Murchison's tables as follows.

The death-rate of typhus-

\begin{tabular}{|c|c|c|c|c|}
\hline \multirow{2}{*}{$\begin{array}{l}\text { Under } \\
\text { From }\end{array}$} & $r$ & ears & is & $17 \cdot 65$ \\
\hline & 5 to 10 & , & is & 7.65 \\
\hline , & 10 to 15 & , & is & 4.95 \\
\hline , & 15 to 22 & ," & is & $4 \cdot 76$ \\
\hline " & 20 to 25 & , & is & 9.5 \\
\hline , & 25 to 30 & , & is & $15 \cdot 15$ \\
\hline , & 60 to 65 & , & is & $55 \cdot 68$ \\
\hline „, & 75 to 80 & , & is & $83 \cdot 33$ \\
\hline
\end{tabular}

(Op. cit., p. 221.)

From a due consideration of the foregoing figures, it is apparent that no safe deduction can be drawn as to the results of treatment from statistics, unless the ages are accurately stated in periods of not less than five years, a precaution not usually adopted by those who have written on this question. Herein consists the doubtful value of the conclusions at which Dr. Gairdner has arrived in his otherwise excellent paper on the Treatment of Typhus without Stimulants, published in the Lancet, March 12th, 1864, and which has been so ably criticised by Dr. Kennedy of Dublin in the same journal of January 7th, 1865.

Having thus endeavoured to point out the difficulties met with in the written history of fever, I proceed to show what may be learnt from actual observation at the bedside, laying aside theory and hypothesis, and reading from the book of nature. As the term fever has such a wide application in the practice of medicine, it is necessary to lay down some limitation in order that it may be clearly understood what disease it is that we propose to discuss. The continued fevers of this country as described by authors of the present day, are: 1 , simple continued fever-the synocha, or short inflammatory fever of Cullen, which usually runs but a short course, generally terminates favourably, and scarcely requiring more than very ordinary attention; 2, typhus fever; 3 , typhoid fever; 4, relapsing fever.

For practical purposes, it will be convenient to take typhus as a type of the rest, and to that form of fever the following observations will be specially applicable.

In this, as in all other diseases, the soundest basis for rational treatment is a clear and definite knowledge of the pathology of the disorder we have to deal with. We therefore proceed to inquire, what is fever? The differing opinions as to its nature may be divided into two great classes ; first, those which consider fever a primary disease, and secondly, those which consider fever to have no existence as such, but regard it as the aggragated symptoms or the consequence of some local or structural lesions. The first of these two theories is that which is perhaps now most generally adopted, ard $\mathrm{d}$ which seems to me the true explanation of the natufe of fever. That the second is untenable may, I think, be satisfactory demonstrated by reference to clinical records. I will endeavour to illustrate this point by relating two well marked examples, taken from the clinical lectures of Dr. Corrigan. The graphic description of what he observed in the Hardwick Hospital, has no doubt been verified in the experience of most practitioners who have seen much of fever.

"A man is seen comatose, lying on his back, with muttering delirium, sordes on his tongue and teeth. pulse 132, skin thickly maculated, with great prostration of strength, involuntary stools, and sensibility so much diminished, that the eyelids remained immoveable, and the bladder had ceased to act. A crisis took place, and in less than two days, of all the above alarming symptoms, debility alone remained; the pulse became regular, the tongue clean, the sensibility natural, sleep returned, and not a trace or symptom of local or structural alteration was discoverable.

"It cannot be supposed that, in such a case, structural disease or local inflammation of several days' duration sufficient to produce this aggregate of alarming symptoms, could have so suddenly vanished."

The second case affords still more decisive evidence on the point in question.

"A man, aged 54, admitted on the 27th February. He was in a state of great debility, and wandering in mind; his tongue dry and brown; pulse thready, 130 ; skin hot, dry, and pungent ; thickly maculated; little or no sleep. On the 2nd of March, debility was greater, and stools passed under him. He still continued to sink, but without any complication. The pulse became scarcely perceptible at the wrist. Wine and stimulants failed to rouse him; and he died on the 10th March, the eleventh day after admission. The most careful post mortem examination failed to discover any local disease."

If the foregoing examples are truthful records of what continually occurs in the clinical history of fever, the conclusion appears inevitable, that fever is to be considered as a primary disease of function, having an existence independent of, and capable of proving fatal without, any local or structural lesion. In order to complete our definition of fever, we proceed to inquire what the functional lesion or lesions are which constitute its essential character; and, if we seek to determine this question from actual observation at the bedside, we see that, in a case of typhus fever, all the most important vital functions are simultaneously deranged. To present this more clearly, I have tabulated the symptoms of fever as follows, in accordance with the views of Dr. Corrigan, as taught in his Clinical Lectures already quoted.

Component Parts of a Case of Typhus Fever: Simultaneous Derangement of all the most important Vital Functions.

A. Derangement of the cerebro-spinal functions; or the lesion of innervation.

$B$. Derangement of the primary nutritive functions, including assimilation, secretion, and excretion; or the lesion of nutrition.

C. Derangement of the circulatory functions; or the lesion of circulation.

a. Of the heart and large vessels.

$b$. Of the capillary system.

Indicated by prostration, want of sleep, delirium, stupor, coma subsultus, involuntary stools, retention of urine.

Want of appetite : thirst, and subsequent loss of desire for fluids; tongue dry or coated ; skin harsh and dry; constipation or diarrhcea; tympanitis; urine scanty and cloudy.

The pulses of the wrist and heart.

Lividity of surface; suffusion of conjunctiva : maculæ; petechiæ; proclivity to sloughing of integuments-bed-sores ; hypostatic congestion of in ternal organs. 
The lesions here set down are to be regarded not merely as symptoms, but as component parts of the disease-deranged functions, which are to be treated by appropriate remedies. It should also be borne in mind that, although it is simultaneous derangement of the great primary vital functions which constitutes a case of fever, yet the proportions which these derangements bear to one another may not be exactly alike in any two individuals; and hence the definition which applies accurately to one case may not suit a second. Each case of fever will derive its distinguishing character from the function which presents the most marked deviation from health. In one case, the circulation is not much disturbed; but there may be great derangement of the function of innervation. In a second case, the cerebral and spinal system and intellect are scarcely at all involved; but the circulation sinks rapidly. In a third case, the function of nutrition, including secretion and excretion, is arrested or unduly excited; while, in a fourth, the nervous, circulatory, and nutritive functions may all be overwhelmed together. It is further obvious that, from the intimate relation and dependence existing between these several fanctions, one cannot be involved to a great extent, or for a continuance, without more or less implicating the other two. It is easy to understand how this simultaneous lesion of so many important functions must soon terminate fatally; one evennamely, that of the cerebral system-is sufficient of itself to cause death, as exemplified in delirium tremens.

This view of the nature of fever, if correct, affords an explanation of those artificial divisions of the disease into brain-fever, nervous fever, gastric or bilious fever, etc. It appears to me to divest the subject of much of the difficulty and obscurity in which students and practitioners must often have found themselves involved in attempting to reconcile the diversity and confliction of opinion which are encountered in perusing the voluminous treatises of fever in which the literature of our profession abounds.

I would here observe, that the foregoing observations may not be considered strictly applicable to typhoid fever, or, as it has been sometimes designated, in accordance with its special pathology, acute follicular enteritis; as, in this form of fever, there is very constantly if not invariably found to exist organic lesion of Peyer's glands, apparently favouring the opinion that in this particular disease the general symptoms are altogether dependent upon local organic lesion. I am not disposed to adopt this view, but believe that the apparent exceptional character of typhoid fever in this respect admits of such an explanation as to bring it fairly within the definition of fever already given. If time permitted, it might, I think, be shown that the ulceration of Peyer's glands, so often found in typhoid fever, is a secondary complication of the primary functional disorder; for it must not be forgotten that, in defining fever to be essentially and primarily a disease of function only, I do not ignore the important consideration that, in the course of a case of fever, organic lesions of a serious nature may occur, in many instances as the direct result of deranged function.

A retrospect of the foregoing analysis of fever gives us a key to the rational treatment of the disease, enabling us to a great extent to reconcile modes of treatment which, although widely different as to the precise remedies employed, may nevertheless each be rationally directed towards the fulfilment of the particular indications which may be present in a given case, and explaining how much that has hitherto seemed vague and conflicting may in reality be re- conciled with fixed principles; and how it is that writers at different epochs may recommend even opposite treatment, and yet be correct in their observations and their practice.

Sydenham has pointed out that the type of fever is frequently changing, and that an acquaintance with the epidemic constitution of the time being is most important. He also adds that, when the type changed, he was frequently very unsuccessful in his practice until observation made him acquainted with the new type. Now, referring to the view we have adopted, it will be seen that the type of the fever can always be discovered by a careful analysis of the functions affected, the type being determined by the function most affected; and local disease, should it arise as a complication, will most frequently occur in the organs principally connected with the performance of that vital function, whatever it may be. Whether, therefore, we have to consider the type of a particular epidemic, or that of a special case, or whether it be a question of constitutional idiosyncrasy or the particular stage of the disease, we have fixed and unerring principles to guide us. No particular line of treatment can be laid down as appropriate in fever; but each lesion of function must be met by its appropriate remedy, to be regulated in application and degree by the extent of the lesion of function which it is intended to relieve.

In the practical application of the foregoing principles, it will be convenient to consider the lesions in order.

First, we take a case where the function most implicated is the cerebro-spinal-a lesion which, if long: continued, terminates in death, either from the direct effect upon itself, or from implication of the functions of organic life. It is manifested by want of sleep or natural rest of the system, which, if extended over a few consecutive nights, is followed by delirium, coma, nervous exhaustion, and perhaps death. This want of sleep is to be regarded not as a mere symptom, but as a part of the disease, a lesion of function of the most serious consequence, and which, if continued, will cause death. Bearing this in mind, we avoid the dangerous error committed by some practitioners (of whom the late Dr. Clutterbuck was the representative), of considering the essence of continued fever to be inflammation of the brain, requiring active depletion for its remedy. Our efforts are directed to the regulation of a derangement of function, and not to the restoration of structural change. If want of sleep be the leading indication, rational means are to be adopted to meet it, by keeping the patient as quiet as possible, not darkening the room too much by day, and thus maintaining the natural alternation of day and night. Cold in some form is applied to the shaven scalp; and, if requisite, an anodyne administered at bedtime. As to the particular form of anodyne which is to be preferred, considerable difference of opinion exists. I have found in my own practice the tincture of henbane, in doses of two drachms, as recommended by Dr. Corrigan, frequently procure sleep; and, when it fails to produce the desired effect, it does not, so far as I am aware, give rise to the injurious effects which sometimes follow the use of opium, to which there is this objection, that it has a tendency to increase the derangement of the function of nutrition; and, in those cases where the digestive organs are much implicated, it is wholly inadmissible. In Dr. Watson's Lectures, the following significant observation of $\mathrm{Dr}$. Latham is quoted. "I have certainly seen," says he, "twenty minims of laudanum produce tranquil sleep, from which the patient has awoke quite a new man; but I have also seen the same quantity produce a fatal coma, from which he has never roused. 
Now," continues Dr. Latham, "since it is a fearful thing to strike a heavy blow in the dark, where the alternative is of such magnitude, it is safest and best to administer a small dose at short intervals." But surely it is better and safer still to select another remedy, which experience has shown will fulfil the indication with perhaps certainty, and with infinitely less risk-probably, indeed, without any risk at all, even when given in full doses. At the same time, I would by no means venture to assert that opium is to be altogether dispensed with in the treatment of fever, but would urge that it should be employed with extreme discrimination and caution. Where other means fail to procure sleep, four or six leeches may be applied to the temples, as recommended by Dr. Corrigan, as an anodyne, upon which he places more reliance than upon any other, and this even when the pulse is weaker than natural; for, as he observes, we need not fear to take a small quantity of blood from the capillaries, for they very soon accommodate themselves to the trifling loss, and the general effect upon the system is scarcely felt; besides which, we can, quite consistently with our principles, give stimulants to support the general circulation, while we are relieving local congestion by moderate depletion from the capillaries.

The internal administration of chloroform has been recommended by the author just quoted; but I cannot from personal observation confirm its efficacy. I have met with cases in which this lesion of innervation, indicated by persistent restlessness and loss of sleep, has resisted every means that could be suggested, and which have invariably proved fatal. In such cases, although we may be able to meet the lesion of circulation by stimulants, yet, unless we can at the same time regulate this lesion of innervation by inducing sleep, the patient is sure to die. It is probable that, from a want of due consideration of this circumstance, the apparent failure of the stimulating mode of treatment has often been misinterpreted.

If stupor or coma come on, or be threatened, blisters may be applied as a means of rousing the patient. A very convenient mode of applying them to the scalp is in broad strips across the top of the head, instead of in the circular form commonly used, which does not lie so flat, or keep on so well.

One of the indications of the lesion of innervation is subsultus tendinum, to be treated on the principles already laid down. Whether this peculiar condition of the nervous system is purely a lesion of function, or whether it depends upon congestion or structural alteration of the cerebro-spinal system, has not been determined. As collateral evidence in favour of the view that the general symptoms of fever are independent of structural lesion, I may here quote the words of Dr. Watson, who, in speaking of the cause of coma, remarks : " Physicians have diligently attempted its solution by examining the dead brain. I cannot tell you," he adds, "how often I have looked, and looked in vain, for some palpable disorganisation, or some effusion implying pressure." ( $O p$. cit.)

We next proceed to consider the lesion of the elementary function of nutrition, upon which both animal and vegetable vitality mainly depends, including assimilation, secretion, and excretion. The symptoms are, loss of appetite, first for food, and afterwards loss of desire for fluid, which, in severe cases, succeeds to the thirst which exists in the earlier period of the disease, and is an aggravated degree of the same lesion. The tongue is dry or coated; sordes on the teeth ; harsh, dry, and hot skin ; derangement of the alvine discharges; constipation or diarrhoea; tympanitis, etc.; and morbid state of the urine. It is scarcely necessary to occupy time in dwelling upon the obvious remedies by which these indications ane to be met; but I may venture to allude to some important points.

First, as to the use of purgatives : these, as already noticed, should be used very sparingly, and, when required, should always be of a mild character. Enemata may often be advantageously substituted. Then as to mercury : in cases where there is a dry coated tongue, absence of thirst, urine cloudy and of low specific gravity, small doses of a mild mercurial, such as blue pill or grey powder, will generally be most useful in restoring the deranged function of nutrition; and, in combination with the alteratives, diuretics may sometimes be advantageously combined.

In this condition of a more or less complete arrest of the function of digestion, we must use much caution not to overload the stomach with nutriment of any kind. Milk, beef-tea, and wine or brandy, in judiciously regulated quantities, guided to a great extent by the desire for them evinced by the patient, is generally all that is required, or that, in fact, can be given without danger of causing still further derangement of the digestive and assimilative functions. As, together with other arrested functions, that of the skin is a prominent symptom, indicated by the dry harsh feeling communicated to the hand when applied to the body of a fever-patient, we might, $a$ priori, conclude that diaphoretics are indicated, and that a renewal of perspiration might be considered a favourable symptom. So far, however, is this from being the case, that crisis by perspiration, as has already been noticed, is much to be dreaded in typhus. It is not sufficient to abstain from giving diaphoretics, but we must also avoid over-heating the patient in any way. I have known a case of typhus which was progressing favourably, and where recovery might have been confidently predicted, but where, from the obstinacy of the patient and his friends, in opposition to the directions of his medical attendant, blankets and bedclothes were heaped upon him, and a copious perspiration, which ended fatally, was the result. As Dr. Corrigan has emphatically remarked: " Popular knowledge, or rather ignorance, confounds maculated or typhus fever with synocha or short inflammatory fever; but the copious perspiration that will cure in the latter will kill in the former."

The next lesion we have to consider is that of the function of the circulation, of that of the heart and large vessels, as indicated by the pulse at the wrist and the impulse of the heart; and of the capillary system, shown by lividity of the surface, injection of the conjunctivæ, maculæ or petechiæ, and proclivity in the integuments to superficial sloughs from pressure. These indications, it is to be borne in mind, are not mere symptoms, or local derangements only, but external indications of the state of the circulatory system as a whole; so that we have to combat a lesion, not of a particular organ or structure, but of a function universally diffused through the whole system. The degree of derangement of the capillary circulation, as shown on the surface by the extent and colour of the macula, is an indication of the condition of the same function throughout the system. Thus rose-coloured macula indicate activity of the capillaries, and are, therefore, more favourable than the darker coloured spots, which are the result of an enfeebled circulation. Petechiæ, or effusions of blood from the capillaries, are signs of greater danger still. The treatment appropriate to this particular lesion is so obvious, that it will at once be anticipated that stimulation and support are the only means upon which we can rely. The best remedy, undoubtedly, 
is alcohol in some form or other. Here is the solution of the much contested question as to how and when stimulants are to be given in fever. Alcohol is the specitic remedy for lesion of the function of circalation involving the cardiac and the capillary systems; so that the state of the pulse and the changes in the colour of the maculæ are the symptoms by which we judge as to the necessity of continuing, decreasing, or augmenting its dose.

If this lesion of the circulation be that of depressed action, alcohol must be given, whatever may be the derangement of the other functions. Consequently, whether there be delirium or not, whether the abdomen be flaccid or tympanitic, whether the tongue be dry or moist, clean or coated, whether it be the first, second, or third week of the fever, are conditions which have no influence whatever in deciding the question. The amount of depression as indicated by the lesion of circulation, modified, of course, by the age, constitutional powers, and previous habits of the patient, are the only circumstances which have to be considered in reference to the use of stimulants. Other forms of stimulants may be sometimes given, of which probably ammonia is the most useful; and here we may remark, that practical experience is at variance with the theory that typhus poison is a compound of ammonia.

It is interesting to notice, that the treatment of typhus by stimulation is by no means a new doctrine. Dr. Trotter, who wrote in 1794, and whose graphic description of the disease shows him to have been a practitioner of much observation, was so impressed with the value of stimulants in typhus, that he sums up his account of the disease with this motto :

"Nil desperandum, Baccho duce et auspice Baccho."

His sagacity had also detected the fallacy of trusting to specifics, as the following brief quotation will show. "Of a febrifuge power residing in antimony, our observation and experience do not enable us to speak in confirmation; nor do we suppose that such a power is to be found in any other medicine whatever." (Medicina Nautica, passim.)

Dr. Stokes of Dublin, writing in 1839, says: “ $I$ feel certain, humiliating though the confession may be, that the fear of stimulants in fever, with which I was imbued, was the means of my losing many patients whose lives would have been saved, had I trusted less to the doctrine of inflammation and more to the lessons of experience given us by men who wrote before the times of Bichat and Hunter." (Dublin Journal of Medical Science, March 1839.)

If the view thus taken of fever be correct, it explains how combinations of remedies may be regarded in those cases where several functions are simultaneously deranged, and that the treatment is to be varied according to the function chiefly affected, and the degree to which it is involved. Thus leeching, cold to the head, or hyoscyamus, may be requisite for lesion of the cerebro-spinal system, mercury for the derangement of the nutritive function, and alcohol for failure of the circulation. So that also the details of treatment may not be alike in any two cases of fever, yet the principles upon which they are founded are fixed.

From this it appears conclusive that, as before mentioned, fever is a disease for which there can be no specific. In the words of Dr. Corrigan, we may conclude: "To suppose there can be any such thing as a specific in fever, would be to claim that the same remedy had equal and opposite powers; that it would highly stimulate the function of circulation in one case and depress it in another; that it would calm dow an excited brain into sleep, and equally restore it from coma to wakefulness ; that it was a remedy of universal power, equally applicable to lesions of all functions, and to such lesions whether arising from increased action, debility, or mere excitement."

\section{ON PUERPERAL FEVER.}

By T. SNow BEck, M.D.Lond., F.R.S., Member of the Royal College of Physicians, London.

[Read before the Obstetrical Society of London, February 1st, 1865.] [Continued.from p. 431.]

Ar first view, these cases present little difference from many others which have been recorded; but, on closer examination, I think they point to two important facts: the pervious condition of the uterine sinuses, by reason of the want of a firm and persistent contraction of the uterus ; and the absence of all inflammatory product, save that observed on the peritoneum. They thus appear to give a decided negative to the opinion that puerperal fever was caused by uterine phlebitis, lymphangitis, endometritis, metritis, or other similar inflammatory affection of the uterine organs. For, with the exception of the inflammation and exudation of the peritoneum, no product of inflammation was any where discovered, after a careful and even microscopical examination of the various tissues which compose the uterus. All of these tissues were in a perfectly healthy state. And I feel considerable confidence in this result, as repeated dissections and examinations of the gravid uterus have made me familiar with the healthy appearance of the tissues which enter into its formation.

But inflammation of the peritoneum, with copious exudation into its cavity, have been long recognised as the morbid appearances most usually observed in this disease. I am not aware, however, of any case where the uterus has been so fully examined, and wherein all the tissues were found perfectly healthy; especially when the woman had survived a period sufficiently long after the commencement of the attack for any inflammatory process to become developed, had this been an essential part of the disease. The total absence of any sign of inflammatory product in the uterus itzelf, whilst the symptoms during life so well characterised the disease, appears to show that the disease has some other cause than these inflammations. The result of the examination of the uterus, moreover, is opposed to some doctrines supported by the highest authority, and furnishes evidences which are contradictory to the opinions expressed by Professor Cruveilhier and supported by Dr. Robert Ferguson. In the opinion of these emi-
nent observers, the internal surface of the uterus is compared to a vast solution of continuity, the muscular tissue being every where exposed; and the gaping orifices of the sinuses are also compared to the open-mouthed vessels of an amputated limb. But the inner surface of the uterus was found every where covered by a soft membrane, which contained all the microscopic elements of the usual mucous membrane. Numerous shreds extended from the inner surface, and gave it the appearance as if the inner portion of its thickness had been torn away. Still the muscular tissue was every where covered by a soft membrane, the surface of which was further covered by the usual red mucous discharge which follows childbirth. Some slight exception must, however, be made on account of that portion to which the placenta had been attached. The membrane was here thicker and firmer than elsewhere, and distinct fibrous tissue entered into its formation. It appeared, in fact, as if a very thin portion of the pla- 